\title{
INFINITELY MANY RADIALLY SYMMETRIC SOLUTIONS TO A SUPERLINEAR DIRICHLET PROBLEM IN A BALL
}

\author{
ALFONSO CASTRO AND ALEXANDRA KUREPA
}

\begin{abstract}
In this paper we show that a radially symmetric superlinear Dirichlet problem in a ball has infinitely many solutions. This result is obtained even in cases of rapidly growing nonlinearities, that is, when the growth of the nonlinearity surpasses the critical exponent of the Sobolev embedding theorem. Our methods rely on the energy analysis and the phase-plane angle analysis of the solutions for the associated singular ordinary differential equation.
\end{abstract}

1. Introduction. Over the last two decades considerable progress has been made in the study of superlinear boundary value problems, such as:

$$
\begin{cases}\Delta u+g(u)=q(x), & x \in \Omega, \\ u=0, & x \in \delta \Omega,\end{cases}
$$

where $\Omega$ is a bounded region in $\mathbf{R}^{N}, \Delta$ is the Laplacian operator, $g: \mathbf{R} \rightarrow \mathbf{R}$ is a continuous function, $q \in L^{2}(\Omega)$, and

$$
\lim _{|u| \rightarrow \infty}(g(u) / u)=\infty
$$

The main goal has been to identify the conditions on $\Omega, g, q$ under which (1.1)(1.2) has infinitely many solutions. Most of the results have been obtained using variational methods. In order to get solutions of (1.1) as critical points of a certain functional $J$ defined in the Sobolev space $\stackrel{\circ}{H}^{1}(\Omega)$ (see Adams $[\mathbf{1}]$ ), it has become customary to assume that $g$ satisfies the growth condition that ensures $J$ to be well defined. For general bounded regions, if $g$ is odd and satisfies suitable growth conditions, the existence of infinitely many solutions was obtained simultaneously and independently by Bahri and Berestycki [2] and Struwe [9]. Their results were later extended by Rabinowitz [8] and Bahri and Lions [3] . The only cases known to us where infinitely many solutions are obtained for $g$ growing up to, but excluding, the limit exponent in the Sobolev space are those of Struwe [10]. In this paper we not only get an extension of those results, but we also get cases where the growth of $g$ surpasses the Sobolev inequality growth. Indeed, our proofs show that for radially symmetric solutions of (1.1) the growth condition depends on the sign of the value of the solution at 0 . Actually our results suggest that it should be sufficient to have the growth conditions only for $u>0$ or for $u<0$.

Received by the editors May 1, 1986. Presented to the AMS at the 791 meeting in Denton, Texas.

1980 Mathematics Subject Classification (1985 Revision). Primary 35J65, 34A10.

Key words and phrases. Superlinear Dirichlet problem, radially symmetric solution, singular ordinary differential equations, phase-plane analysis, growth condition, rapidly growing nonlinearities. 
We study problem (1.1) when $\Omega$ is the ball of radius $T$ in $\mathbf{R}^{N}$, centered at the origin, and

$$
q(x)=p(\|x\|)
$$

is a radially symmetric function. For the sake of simplicity of the proofs we assume, without loss of generality, that

$$
p=L^{\infty}(\Omega), g(0)=0, \text { and } g \text { is strictly increasing. }
$$

In order to state our main result we introduce the following notation:

$$
\begin{gathered}
L(k, u)=N G(k u)-((N-2) / 2) u\left(g(u)+5\|p\|_{\infty} \operatorname{sgn}(u)\right) \\
L_{ \pm}(k)=\lim _{u \rightarrow \pm \infty} L(k, u)(u / g(u))^{N / 2} \\
F(m, u)=(u / g(u))^{N+\rho-1} \cdot G(k u)+m
\end{gathered}
$$

where $G(u)=\int_{0}^{u} g(v) d v, k \in(0,1], \rho>0$, and $m \in \mathbf{R}$.

Our main result is

THEOREM A. Suppose $g$ is locally Lipschitzian and (1.2)-(1.3) hold. If

(i) $L(1, u)$ is bounded below and $L_{+}(k)=\infty$ (respectively $\left.L_{-}(k)=\infty\right)$ for some $k \in(0,1)$, or

(ii) $F(m, u) \rightarrow \infty$ as $u \rightarrow \infty$ (respectively $F(m, u) \rightarrow \infty$ as $u \rightarrow-\infty$ ) for any $m \in \mathbf{R}$,

then (1.1) has infinitely many radially symmetric solutions with $u(0)>0$ (respectively $u(0)<0)$ ).

Theorem $\mathrm{A}$ includes the results of Struwe [10] since hypothesis (i) is satisfied for $|g(u)| \leq A|u|^{\omega}+B, A, B \in \mathbf{R}, 1<\omega<(N+2) /(N-2)$. Not only that, condition (i) allows the case $g(u)=u^{(N+2) /(N-2)}+u^{\omega}$ for either $u<0$ or $u>0$. Also, if $|g(u)|<A u^{\sigma}, A \in \mathbf{R}, 1<\sigma<N /(N-2)$, then condition (ii) holds without any growth restriction on $g(u)$ for $u<0$. Hence, cases such as $g(u)=$ $u^{\kappa}-e^{-u^{3}}(N=2, \kappa$ odd) are encompassed by hypothesis (i). These results, to the best of our knowledge, have not been obtained before. Moreover, since our method is not variational we do not have to use compactness arguments of Palais-Smale type. Therefore cases such as $g(u)=u \cdot \log (|u|),|u|>1$, satisfy the hypothesis of Theorem $A$, but not the hypothesis required in the work of others (see for example $\left.\left[2,(6.3) ; 3 ; 8,\left(\mathrm{p}_{3}\right) ; 10,(4)\right]\right)$. Another advantage of our method is that $p$ can be allowed to depend on $\left(\|x\|, u, u^{\prime}\right)$ as long as $p\left(\|x\|, u, u^{\prime}\right)$ is uniformly bounded. This is not possible when using variational arguments (see $[\mathbf{2}, \mathbf{3}, \mathbf{8}, \mathbf{9}, \mathbf{1 0}]$ ).

Our proofs rely on the study of the singular initial value problem

$$
\begin{aligned}
u^{\prime \prime}+\frac{n}{t} u^{\prime}+g(u) & =p(t), \quad t \in[0, T], \\
u(0) & =d, \\
u^{\prime}(0) & =0,
\end{aligned}
$$

where $n=N-1$, and $d$ is an arbitrary real number. A simple argument based on the contraction mapping principle and Lemma 2.1 shows that problem (1.8) has a unique solution $u(t, d)$ on the interval $[0, \infty)$ depending continuously on $d$. In $\S 2$ we analyze the energy of the corresponding solutions, i.e. we analyze the function

$$
E(t, d)=\left(u^{\prime}(t, d)\right)^{2} / 2+G(u(t, d)) .
$$


Since radially symmetric solutions of (1.1) are the solutions of (1.8) satisfying

$$
u(T, d)=0
$$

we use the "shooting" method. In order to do so we estimate from below the value of $t_{0}>0$ such that the solution of (1.8) satisfies

$$
u\left(t_{0}, d\right)=k d, \quad k \in(0,1) .
$$

Combining this estimate, hypotheses (1.2)-(1.3), and an argument resembling the one used in the proof of Pohozaev's identity we show that

$$
\lim _{d \rightarrow+\infty} E(t, d)=\infty \quad\left(\text { respectively } \lim _{d \rightarrow-\infty} E(t, d)=\infty\right),
$$

uniformly for $t \in[0, T]$.

The rest of the proof is based on the phase plane analysis. From (1.12) it follows that in the $\left(u, u^{\prime}\right)$ plane for $d$ sufficiently large a continuous argument function $\theta(t, d)$ can be defined so that $\theta(0, d)=0$. Imitating the proof used by Castro and Lazer [4] in $\S 3$ we show that

$$
\lim _{d \rightarrow \infty} \theta(T, d)=\infty
$$

Thus, by the intermediate value theorem we have the existence of infinitely many solutions for (1.10), therefore infinitely many radially symmetric solutions for (1.1)

In a forthcoming paper we treat the jumping nonlinearity case extending the work of Castro and Shivaji [5].

2. Energy analysis. Throughout this paper we assume that (1.4) holds. The continuous dependence of $u$ and $u^{\prime}$ in $(t, d)$ can be obtained by considering the operator

$$
(u, d) \rightarrow d+\int_{0}^{t} r^{-n} \int_{0}^{r} s^{n}(p(s)-g(u(s))) d s d r
$$

in the complete metric space $(C[0, \varepsilon]) \times\left[d^{\prime}-\varepsilon, d^{\prime}+\varepsilon\right]$, and showing that for each $d \in \mathbf{R}$ this operator defines a contraction in $u$.

LEMMA 2.1. Every solution to (1.8) is defined for all $t \in[0, \infty)$.

PROOF. Suppose that for some increasing sequence $t_{n} \rightarrow \bar{t} \in \mathbf{R}$ we have

$$
\lim _{n \rightarrow \infty}\left(u^{2}\left(t_{n}, d\right)+\left(u^{\prime}\left(t_{n}, d\right)\right)^{2}\right) \rightarrow \infty .
$$

If $\left(u^{\prime}\left(t_{n}, d\right)\right)^{2}$ does not tend to infinity, then by the mean value theorem a new increasing sequence $t_{n}^{\prime} \rightarrow \bar{t}$ can be found so that $\left(u^{\prime}\left(t_{n}^{\prime}, d\right)\right)^{2} \rightarrow \infty$. Thus, without loss of generality, we can assume $\left(u^{\prime}\left(t_{n}, d\right)\right)^{2} \rightarrow \infty$. Also, since (1.4) holds, we have $G \geq 0$. Hence

$$
\lim _{n \rightarrow \infty} E\left(t_{n}, d\right)=\infty
$$

On the other hand we have

$$
\begin{aligned}
E^{\prime}(t, d) & =u^{\prime}(t, d) p(t)-\frac{n}{t}\left(u^{\prime}(t, d)\right)^{2} \\
& \leq\left|u^{\prime}(t, d)\right|\|p\|_{\infty} \leq \sqrt{2}\|p\|_{\infty} \sqrt{E(t, d)}
\end{aligned}
$$


Hence

$$
E(t, d) \leq\left(\sqrt{E(0, d)}+(\sqrt{2} / 2)\|p\|_{\infty} t\right)^{2}
$$

which contradicts (2.1) and thus the lemma is proven.

For $k \in(0,1)$ and $d>0$ with $|g(d)|>\|p\|_{\infty}$ let $t_{0}:=t_{0}(k, d)$ be such that $d \geq u(t, d)>k d$ for all $t \in\left[0, t_{0}\right)$ and $u\left(t_{0}, d\right)=k d$. Since we are assuming $g$ to be an increasing function we have

$$
u^{\prime}(t, d)=t^{-n} \int_{0}^{t} r^{n}(p(r)-g(u(r, d))) d r \geq\left(-\|p\|_{\infty}-g(d)\right) t /(n+1) .
$$

Hence, integrating over $\left[0, t_{0}\right]$ we obtain

$$
t_{0} \geq\left(2(n+1)(1-k) d /\left(\|p\|_{\infty}+g(d)\right)\right)^{1 / 2} .
$$

Similar arguments show that if $d<0$ and $|g(d)|>\|p\|_{\infty}$ then (2.2) also holds. Now we are ready to prove

LEMMA 2.2. If $L(1, u)$ is bounded below and for some $k \in(0,1), L_{+}(k)=\infty$ (respectively $\left.L_{-}(k)=\infty\right)$, then

$$
\lim _{d \rightarrow \infty} E(t, d)=\infty \quad\left(\text { respectively } \lim _{d \rightarrow-\infty} E(t, d)=\infty\right)
$$

uniformly for $t \in[0, T]$.

ProOF. Let $v(x)=u(\|x\|), x \in \Omega$. Since $u$ satisfies (1.8) we have

$$
\begin{gathered}
\Delta v+g(v)=p(\|x\|), \quad x \in \Omega, \\
v(0)=d, \quad \nabla v(0)=0 .
\end{gathered}
$$

The arguments that follow are inspired by Pohozaev's identity. Throughout the proof $c$ denotes various positive constants depending on $\left(N,\|p\|_{\infty}, g\right)$. An elementary calculation shows that

$$
\begin{aligned}
(\Delta v)(x \cdot \nabla v)= & \frac{N-2}{2}\|\nabla v\|^{2}+\sum_{i=1}^{N}\left(\sum_{j=1}^{N} x_{j} v_{x_{i}} v_{x_{j}}\right)_{x_{i}} \\
& -\frac{1}{2} \sum_{j=1}^{N}\left(\sum_{i=1}^{N} x_{j} v_{x_{i}}^{2}\right)_{x_{j}}, \\
(g(v)-p(\|x\|))(x \cdot \nabla v)= & \left(\sum_{j=1}^{N}\left(x_{j} G(v)\right)_{x_{j}}\right)-N G(v)-p(\|x\|)(x \cdot \nabla v),
\end{aligned}
$$

where the subindex $x_{k}$ denotes the partial derivative with respect to the variable $x_{k}$. 
Multiplying (2.3) by $x \cdot \nabla v$, integrating over $\Omega_{\varepsilon}=\{x \in \Omega$; $\|x\|<\varepsilon\}, t_{0} \leq \varepsilon \leq T$ (see (2.2), (2.5)), and using the divergence theorem we have

$$
\begin{gathered}
\int_{\Omega_{\varepsilon}}\left[\frac{N-2}{2}\|\nabla v\|^{2}-N G(v)-p(\|x\|)(x \cdot \nabla v)\right] d x \\
=\int_{\delta \Omega_{\varepsilon}}\left[\sum_{j=1}^{N} \nu_{j}\left(\frac{1}{2} \sum_{i=1}^{N} x_{j} v_{x_{i}}^{2}-x_{j} G(v)\right)-\sum_{j=1}^{N} \nu_{j}\left(\sum_{i=1}^{N} x_{i} v_{x_{i}} v_{x_{j}}\right)\right] d \sigma \\
=\int_{\delta \Omega_{\varepsilon}}-\left[\sum_{j=1}^{N} \nu_{j}\left(\frac{1}{2} \sum_{i=1}^{N} x_{j} v_{x_{i}}^{2}+x_{j} G(v)\right)\right] d \sigma
\end{gathered}
$$

where $\nu=\left(\nu_{1} \cdots \nu_{N}\right)$ denotes the outward unit normal to $\Omega_{\varepsilon}$ at $x$, and we have used the radial symmetry of $v$.

On the other hand, multiplying (2.3) by $v$ and integrating over $\Omega_{\varepsilon}$ we have

$$
\int_{\Omega_{\varepsilon}}\|\nabla v\|^{2} d x=\int_{\Omega_{\varepsilon}}(g(v)-p(\|x\|)) v d x+\int_{\delta \Omega_{\varepsilon}} v(\nu \cdot \nabla v) d \sigma
$$

Replacing (2.7) in (2.6) we obtain

$$
\begin{aligned}
\int_{\Omega_{\varepsilon}} & \left(N G(v)-\frac{(N-2) g(v) v}{2}\right) d x \\
= & \int_{\delta \Omega_{\varepsilon}}\left[\frac{\varepsilon}{2}\|\nabla v\|^{2}+\varepsilon G(v)+\frac{N-2}{2}(v(\nu \cdot \nabla v))\right] d \sigma \\
& \quad-\int_{\Omega_{\varepsilon}} p(\|x\|)\left((x \cdot \nabla v)+\frac{N-2}{2} v\right) d x
\end{aligned}
$$

Thus, from (2.2), (2.8), and the assumption that $L(1, u)$ is bounded below we have

$$
\left(\frac{d}{g(d)}\right)^{N / 2}\left(N G(k d)-\frac{(N-2) g(d) d}{2}\right)+\left(\varepsilon^{N}-t_{0}^{N}\right) B \leq c E(\varepsilon),
$$

where $B$ is a constant, possibly negative, independent of $d$. Since, in addition, $L_{+}(k)=\infty$, from $(2.9)$ we have

$$
\lim _{d \rightarrow \infty} E(t, d)=\infty
$$

uniformly for $t \in[0, T]$, and this proves Lemma 2.2 .

LEMMA 2.3. If for any $m \in \mathbf{R} F(m, u) \rightarrow \infty$ as $u \rightarrow \infty$ (respectively $F(m, u)$ $\rightarrow \infty$ as $u \rightarrow-\infty)$, then

$$
\lim _{d \rightarrow \infty} E(t, d)=\infty \quad\left(\text { respectively } \lim _{d \rightarrow-\infty} E(t, d)=\infty\right)
$$

uniformly for $t \in[0, T]$.

ProOF. From the definition of $E$ and (1.8) we have

$$
\left(t^{2(n+\rho)} E(t, d)\right)^{\prime} \geq 2(n+\rho) t^{2(n+\rho)-1} G(u(t, d))-\left(t^{2(n+\rho)+1}\|p\|_{\infty}^{2}\right) / 4 \rho .
$$

Integrating over $\left[t_{0}, t\right]$, we infer

$$
E(t, d) \geq T^{-2(n+\rho)}\left[t_{0}^{2(n+\rho)} E\left(t_{0}, d\right)+m\right],
$$


where $m$ is a constant depending on $\left(n, T,\|p\|_{\infty, \rho}\right)$, and we have used the fact that $G \geq 0$. Hence, from (2.2) and (2.10) by the hypothesis of the lemma we obtain the proof.

3. Phase-plane analysis. Let $\left(u(t, d), u^{\prime}(t, d)\right) \neq(0,0)$ for all $t \in[0, \bar{t})$. By defining $r^{2}(t, d)=u^{2}(t, d)+\left(u^{\prime}(t, d)\right)^{2}$ we see that for $d>0$ there exists a unique continuous argument function $\theta(t, d), t \in[0, \bar{t})$, such that

$$
\begin{aligned}
& u(t, d)=r(t, d) \cos \theta(t, d), \\
& u^{\prime}(t, d)=-r(t, d) \sin \theta(t, d), \\
& \theta(0, d)=0 .
\end{aligned}
$$

If $d<0$ the argument function $\psi(t, d)$ is given by

$$
\begin{aligned}
& u(t, d)=-r(t, d) \cos \psi(t, d), \\
& u^{\prime}(t, d)=r(t, d) \sin \psi(t, d), \\
& \psi(0, d)=0 .
\end{aligned}
$$

An elementary calculation shows that

$$
\theta^{\prime}(t, d)=\sin ^{2} \theta(t, d)+\frac{\left(g(u(t, d))+\frac{n}{t} u^{\prime}(t, d)-p(t)\right) \cos \theta(t, d)}{r(t, d)} .
$$

From this formula follows

REMARK 3.1. There exists $R$ such that if $r(t, d) \geq R$ for all $t \in[0, T]$, and $\theta(\bar{t}, d)=(k \pi / 2)$ for some $\bar{t} \in[0, T]$ and a nonnegative integer $k$, then $\theta(t, d)>$ $(k \pi / 2)$ for all $t>\bar{t}$.

PROOF OF THEOREM A. By the continuous dependence of the solutions on the initial conditions it is sufficient to prove that

$$
\lim _{d \rightarrow \infty} \theta(T, d)=\infty \quad\left(\text { respectively } \lim _{d \rightarrow-\infty} \psi(T, d)=\infty\right) .
$$

In order to do so, we show that, given any positive integer $J$, there exists $d_{0}$ such that if $d \geq d_{0}$ (respectively $-d \geq d_{0}$ ), then $\theta(T, d)>J \pi$ (respectively $\psi(T, d)>$ $J \pi)$.

If $x_{0}>0$ and $m\left(x_{0}\right):=\min \left\{(g(x) / x):|x| \geq x_{0}\right\}$, then by (1.2) we have

$$
m\left(x_{0}\right) \rightarrow \infty \text { as } x_{0} \rightarrow \infty \text {. }
$$

Let

$$
\delta \in(0, \min \{\pi / 4, T / 64 n\}),
$$

and $r_{0}$ be such that

$$
\begin{gathered}
8\|p\|_{\infty}<r_{0} \\
16 \delta+2 \pi / \omega\left(r_{0}, \delta\right) \leq 3 T / 4 J \\
\omega\left(r_{0}, \delta\right)>(4 n / T)+\left(2\|p\|_{\infty} / r_{0}\right)
\end{gathered}
$$

where $\omega\left(r_{0}, \delta\right)=m\left(r_{0} \cos \delta\right) \sin ^{2} \delta$.

Since $r(t, d) \rightarrow \infty$ when $E(t, d) \rightarrow \infty$, by Lemmas 2.2 and 2.3 we see that there exists $d_{0}$ such that if $d>d_{0}$, then $r(t, d) \geq r_{0}$ for all $t \in[0, T]$. In particular $\theta(t, d)$ is defined for all $t \in[0, T]$. 
Suppose that $t \geq(T / 4)$ and $\theta(t, d)$ belongs to an interval of the form [ $(j \pi / 2)-$ $\delta,(j \pi / 2)+\delta]$, where $j$ is a nonnegative odd integer. From (3.3) we have

$$
\begin{aligned}
\theta^{\prime}(t, d) & \geq \sin ^{2} \theta(t, d)+\frac{g(u(t, d)) u(t, d)}{r^{2}(t, d)}-\frac{4 n\left|u(t, d) u^{\prime}(t, d)\right|}{\operatorname{Tr}^{2}(t, d)}-\frac{\|p\|_{\infty}}{r_{0}} \\
& \geq \cos ^{2} \delta-\frac{4 n \tan \delta}{T}-\frac{\|p\|_{\infty}}{r_{0}} \geq \frac{1}{4}
\end{aligned}
$$

where we have used (3.6), (3.7), and that $g(u) \cdot u \geq 0$ since (1.4) holds.

On the other hand, if $\theta(t, d)$ belongs to an interval of the form $[(j \pi / 2)+\delta$, $(j+2)(\pi / 2)-\delta$ ] we have

$$
\begin{aligned}
\theta^{\prime}(t, d) & \geq\left(g(u(t, d)) \sin ^{2} \delta\right) /(u(t, d))-(2 n / T)-\left(\|p\|_{\infty} / r_{0}\right) \\
& \geq \omega\left(r_{0}, \delta\right) / 2>0
\end{aligned}
$$

(see (3.9)).

By Remark 3.1 we see that $\theta(T / 4, d)>0$ for all $d \geq d_{0}$. Now, by (3.10) and (3.11) we infer that $\theta(t, d)$ is an increasing function of $t$ on the interval $[T / 4, T]$.

Next we estimate $\theta(T, d)$. Suppose that $|\theta(T / 4, d)-(j \pi / 2)|<\delta$ for some odd positive integer $j$. Since by $(3.10) \theta(t, d)$ cannot remain in the interval $[(j \pi / 2)-$ $\delta,(j \pi / 2)+\delta]$ longer than $8 \delta$, there exists $t_{1} \in(T / 4,(T / 4)+8 \delta)$ such that

$$
\theta\left(t_{1}, d\right)=(j \pi / 2)+\delta
$$

Now, for $t>t_{1}$ with $\theta(s, d) \in[(j \pi / 2)+\delta,(j+2)(\pi / 2)-\delta]$ and for all $s \in\left[t_{1}, t\right]$ from (3.11) we see that $t-t_{1}<(\pi-2 \delta) / \omega\left(r_{0}, \delta\right)$. Thus, there exists $t_{2} \in\left(t_{1}, t_{1}+\right.$ $\left.\left(2(\pi-2 \delta) / \omega\left(r_{0}, \delta\right)\right)\right)$ and

$$
\theta\left(t_{2}, d\right)=(j+2)(\pi / 2)-\delta
$$

Imitating the argument used in establishing the existence of $t_{1}$, we see that there exists $t_{3} \in\left(t_{2}, t_{2}+8 \delta\right)$ such that $\theta\left(t_{3}, d\right)=(j+2)(\pi / 2)+\delta$. Using the properties of $t_{1}$ and $t_{2}$ we obtain

$$
\begin{aligned}
& T / 4<t_{3}<(T / 4)+16 \delta+\left(2(\pi-2 \delta) / \omega\left(r_{0}, \delta\right)\right), \\
& \theta\left(t_{3}, d\right)-\theta(t, d)>\pi .
\end{aligned}
$$

Repeating this argument $J$ times we see that

$$
\theta(\bar{t}, d) \geq J \pi
$$

for some $\bar{t} \in\left[T / 4,(T / 4)+J\left(16 \delta+\left(2(\pi-2 \delta) / \omega\left(r_{0}, \delta\right)\right)\right)\right]$. From (3.15) using (3.8) we get $\bar{t} \leq T$. Since $\theta(t, d)$ is increasing we have proved that

$$
\theta(T, d) \geq J \pi
$$

On the other hand if $|\theta(T / 4, d)-(j \pi / 2)| \geq \delta$, similar arguments lead to the existence of $\bar{t} \in\left[T / 4,(T / 4)+J\left(8 \delta+\left(4(\pi-2 \delta) / \omega\left(r_{0}, \delta\right)\right)\right)\right]$ such that $\theta(\bar{t}, d) \geq J \pi$. Hence,

$$
\theta(T, d) \geq J \pi
$$

From (3.16) and (3.17) follows (3.4). Since the case $d<0$ follows the identical pattern, the theorem is proven. 


\section{REFERENCES}

1. R. Adams, Sobolev spaces, Academic Press, New York, 1975.

2. A. Bahri and H. Berestycki, A perturbation method in critical point theory and applications, Trans. Amer. Math. Soc. 267 (1981), 1-32.

3. A. Bahri and P. L. Lions, Remarques sur la theorie variationnelle des points critiques et applications, C. R. Acad. Sci. Paris 301 (1985), 145-149.

4. A. Castro and A. C. Lazer, On periodic solutions of weakly coupled systems of differential equations, Boll. Un. Mat. Ital. B (5) 18 (1981), 733-742.

5. A. Castro and R. Shivaji, Multiple solutions for a Dirichlet problem with jumping nonlinearities. II, J. Math. Anal. Appl. (to appear).

6. M. J. Esteban, Multiple solutions of semilinear elliptic problems in a ball, J. Differential Equations 57 (1985), 112-137.

7. S. Fucik and V. Lovicar, Periodic solutions of the equation $x^{\prime \prime}(t)+g(x(t))=p(t)$, Casopis Pěst. Mat. 100 (1975), 160-175.

8. P. H. Rabinowitz, Multiple critical points of perturbed symmetric functionals, Trans. Amer. Math. Soc. 272 (1982), 753-769.

9. M. Struwe, Infinitely many critical points for functionals which are not even and applications to superlinear boundary value problems, Manuscripta Math. 32 (1980), 335-364.

10. $ـ$ Superlinear elliptic boundary value problems with rotational symmetry, Arch. Math. 39 (1982), 233-240.

Department of Mathematics, North Texas State University, Denton, Texas 